\title{
The effect of the antioxidant drug "U-74389G" on alkaline phosphatase levels during ischemia reperfusion injury in rats
}

\author{
Constantinos Tsompos ${ }^{\mathrm{a}^{*}}$, Constantinos Panoulis ${ }^{\mathrm{b}}$, Konstantinos Toutouzas ${ }^{\mathrm{c}}$, George Zografos ${ }^{\mathrm{c}}$, Apostolos Papalois ${ }^{\mathrm{d}}$ \\ ${ }^{a}$ Department of Obstetrics and Gynecology, Mesologi County Hospital, Etoloakarnania, Greece \\ ${ }^{b}$ Department of Obstetrics and Gynecology, Aretaieion Hospital, Athens University, Attiki, Greece \\ ${ }^{c}$ Department of Surgery, Ippokrateion General Hospital, Athens University, Attiki, Greece \\ ${ }^{d}$ Director, Exprerimental Research Center ELPEN Pharmaceuticals, S.A.Inc., Co, Greece
}

\section{ARTICLE INFO}

\section{Article History}

Received $\quad 16 / 03 / 2014$

Accepted $\quad 10 / 05 / 2014$

\section{* Correspondence to:}

Constantinos Tsompos

Department of Obstetrics and Gynecology,

Mesologi County Hospital,

Etoloakarnania, Greece

e-mail: Constantinostsompos@yahoo.com

\section{Keywords:}

Alkaline phosphatase levels

Ischemia

Reperfusion

U-74389G

\section{ABSTRACT}

The aim of this experimental study was to examine the effect of the antioxidant drug "U-74389G", on rat model and particularly in a liver ischemia reperfusion (IR) protocol. The beneficial effect or non-effectiveness of that molecule were studied biochemically using mean blood alkaline phosphatase levels (ALP). Forty rats of mean weight 231.875 gr were used in the study. ALP levels were measured 60 min after reperfusion (groups $\mathrm{A}$ and $\mathrm{C}$ ) and 120 min after reperfusion (groups B and D) with administration of the drug U-74389G in groups C and D. U-74389G administration non-significantly increased the ALP levels by $19.76 \% \pm 15.06 \%(\mathrm{p}=0.1784)$, but by $31.91 \% \pm 7.69 \%(\mathrm{p}=0.0001)$ for predicted values. Reperfusion time non-significantly decreased the ALP levels by $25.21 \% \pm 14.83 \%(\mathrm{p}=0.0675)$, also by $6.38 \% \pm 9.30 \%(\mathrm{p}=0.3650)$ for predicted values. However, U-74389G administration and reperfusion time together non-significantly increased the ALP levels by $8.08 \% \pm 9.19 \%(\mathrm{p}=0.3696)$, but also by $17.75 \% \pm 4.79 \%$ $(\mathrm{p}=0.0005)$ for predicted values. This study indicates that $\mathrm{U}-74389 \mathrm{G}$ administration and its interaction with reperfusion time non-significantly increased short-term effects on ALP levels. However, the predicted ALP values adjusted for rats weight, kept significantly increased ones, which stand for the restorating effects of U-74389G, that are slower than it seems.

J. Exp. Clin. Med., 2014; 31:99-102

\section{Introduction}

Tissue ischemia and reperfusion (IR) remain one of the main causes of permanent or transient damage with serious implications on adjacent organs and certainly on patient's health. The use of antioxidant substances has been a research subject for many years. However, even if important progress has been made, satisfactory answers have not been given yet to fundamental questions, such as, how much powerful should an antioxidant be, when should it be administered, and in which dosage. The particularly satisfactory action of the antioxidant U-74389G in tissue protection has been noted in several experiments. Since a careful literature search (PubMed-Medline) was conducted, it was realized that this certain antioxidant has been tried in IR experiments. However, just few relative reports were found, not covering completely this particular matter. Also, a lot of publications addressed trials of other similar molecules of aminosteroids (lazaroids) to which the studied molecule also belongs to. U-74389G (https) or better 21-[4-(2,6-di-1-pyrrolidinyl-4-pyrimidinyl)1-piperazinyl]-pregna-1,4,9(11)-triene-3,20-dione maleate salt1 is an antioxidant which prevents both arachidonic acidinduced and iron-dependent lipid peroxidation. Fenglin et al., (1995) found that it protects against IR injury in animal heart, liver and kidney models. These membrane-associating antioxidants are particularly effective in preventing permeability changes in brain microvascular endothelial cells monolayers.

The aim of this experimental study was to examine the effect of the antioxidant drug "U-74389G" on rat model and particularly in a liver IR protocol. The beneficial effect or non-effectiveness of that molecule were studied by measuring blood alkaline phosphatase levels (ALP). 
Table 1. Weight and ALP levels and Standard deviation of groups

\begin{tabular}{lccc} 
Groups & Variable & Mean & Std. Dev \\
\hline A & Weight & $243 \mathrm{gr}$ & $45.77724 \mathrm{gr}$ \\
& ALP & $131.3 \mathrm{IU} / \mathrm{L}$ & $80.18458 \mathrm{IU} / \mathrm{L}$ \\
B & Weight & $262 \mathrm{gr}$ & $31.10913 \mathrm{gr}$ \\
& ALP & $90.8 \mathrm{IU} / \mathrm{L}$ & $43.3123 \mathrm{IU} / \mathrm{L}$ \\
$\mathrm{C}$ & Weight & $212.5 \mathrm{gr}$ & $17.83411 \mathrm{gr}$ \\
& ALP & $141.9 \mathrm{IU} / \mathrm{L}$ & $25.4709 \mathrm{IU} / \mathrm{L}$ \\
D & Weight & $210 \mathrm{gr}$ & $18.10463 \mathrm{gr}$ \\
& ALP & $125.1 \mathrm{IU} / \mathrm{L}$ & $39.65252 \mathrm{IU} / \mathrm{L}$ \\
\hline
\end{tabular}

Table 2. Statistical significance of mean values difference for groups after statistical paired t test application

\begin{tabular}{lccc} 
DG & Variable & Difference & p-value \\
\hline A-B & Weight & $-19 \mathrm{gr}$ & 0.2423 \\
& ALP & $40.5 \mathrm{IU} / \mathrm{L}$ & 0.0859 \\
A-C & Weight & $30.5 \mathrm{gr}$ & 0.0674 \\
& ALP & $-10.6 \mathrm{IU} / \mathrm{L}$ & 0.6983 \\
A-D & Weight & $33 \mathrm{gr}$ & 0.0673 \\
& ALP & $6.2 \mathrm{IU} / \mathrm{L}$ & 0.8117 \\
B-C & Weight & $49.5 \mathrm{gr}$ & 0.0019 \\
& ALP & $-51.1 \mathrm{IU} / \mathrm{L}$ & 0.0129 \\
B-D & Weight & $52 \mathrm{gr}$ & 0.0009 \\
& ALP & $-34.3 \mathrm{IU} / \mathrm{L}$ & 0.0917 \\
C-D & Weight & $2.5 \mathrm{gr}$ & 0.7390 \\
& ALP & $16.8 \mathrm{IU} / \mathrm{L}$ & 0.3589 \\
\hline
\end{tabular}

\section{Materials and methods}

\section{Animal preparation}

This experimental study was laid out at the Exprerimental Research Center of ELPEN Pharmaceuticals Co. Inc. S.A. at Pikermi, Attiki and by Veterinary Address of East Attiki Prefecture under 3693/12-11-2010 \& 14/10-1-2012 decisions. All settings needed for the study including of consumables, equipment and substances used, were a courtesy of that $\mathrm{S}$. A. Albino female Wistar rats were used in accordance with accepted standards of human animal care. They spent in laboratory 7 days before the experiment with easy access to water and food. The experiment was acute, that is, the animal usage was completed by following experimental set of times without awakening and preservation of the rodents. They were randomly assigned to four experimental groups (10 animals in each group). 1) Ischemia for $45 \mathrm{~min}$ followed by reperfusion for $60 \mathrm{~min}$ (group A). 2) Ischemia for $45 \mathrm{~min}$ followed by reperfusion for $120 \mathrm{~min}$ (group B). 3) Ischemia for $45 \mathrm{~min}$ followed by immediate U-74389G intravenous (IV) administration and reperfusion for $60 \mathrm{~min}$ (group C). 4) Ischemia for $45 \mathrm{~min}$ followed by immediate U-74389G IV administration and reperfusion for $120 \mathrm{~min}$ (group D). The molecule U-74389G dose was $10 \mathrm{mg} / \mathrm{kg}$ body weight of animals.

The experiment was started with prenarcosis and general anesthesia administration in animals. Their electrocardiogram and acidometry were continuously monitored. The inferior aorta was prepared so as its blood flow to be excluded by forceps. After exclusion, the protocol of IR was applied, exactly as described in experimental groups. The molecules were administered at the time of reperfusion, through inferior vena cava after catheterization. The ALP measurement was performed at $60 \mathrm{~min}$ of reperfusion (groups A and C) and 120 min of reperfusion (groups B and D).

Rats underwent general anesthesia by initial intramuscular (IM) administration of $0.5 \mathrm{cc}$ compound, which constituted of $0.25 \mathrm{cc}$ xylazine, $[25 \mathrm{cc}, 20 \mathrm{mg} / \mathrm{cc}$ ] and $0.25 \mathrm{cc}$ ketamine hydrochloride [1000 mg, 100mg/cc, 10cc]. Before initiation of laparotomy, $0.03 \mathrm{cc}$ butorphanol $[10 \mathrm{mg} / \mathrm{cc}, 10 \mathrm{cc}]$ anesthetic agent was administered subcutaneous (SC). Continuous oxygen supply was administered during whole experiment performance. Ischemia was caused by clamping inferior aorta over renal arteries for $45 \mathrm{~min}$ after laparotomic access was achieved. Reperfusion was induced by removing the clamp and reestablishment of inferior aorta patency. Forty (40) female Wistar albino rats were used of mean weight 231.875 gr [Std. Dev: 36.59703 gr], with min weight $\geq 165$ gr and $\max$ weight $\leq 320$ gr. Rats weight could be potentially a confusing factor, e.g. fatter rats to have greater blood ALP levels. This suspicion will be investigated.

\section{Model of ischemia-reperfusion injury}

Control groups: Twenty control rats of mean weight 252.5 gr [Std. Dev: $39.31988 \mathrm{gr}$ ] were subjected to ischemia for 45 min followed by reperfusion.

Group A: Reperfusion which lasted 60 min concerned 10 controls rats of mean weight 243 gr [Std. Dev: 45.77724 gr], mean ALP levels 131.3 IU/L [Std. Dev: 80.18458 IU/L] (Table 1).

Group B: Reperfusion which lasted 120 min concerned 10 controls rats of mean weight 262 gr [Std. Dev: 31.10913 gr], mean ALP levels 90.8 IU/L [Std. Dev: 43.3123 IU/L] (Table 1).

Lazaroid (L) group: Twenty rats of mean weight $211.25 \mathrm{gr}$ [Std. Dev: $17.53755 \mathrm{gr}$ ] suffered by ischemia for $45 \mathrm{~min}$ followed by reperfusion in the beginning of which $10 \mathrm{mg}$ U-74389G / kg body weight were IV administered.

Group C: Reperfusion which lasted 60 min concerned $10 \mathrm{~L}$ rats of mean weight $212.5 \mathrm{gr}$ [Std. Dev: $17.83411 \mathrm{gr}$ ], mean ALP levels 141.9 IU/L [Std. Dev: 25.4709 IU/L] (Table 1).

Group D: Reperfusion which lasted 120 min concerned 10 L rats of mean weight $210 \mathrm{gr}$ [Std. Dev: $18.10463 \mathrm{gr}$ ], mean ALP levels 125.1 IU/L [Std. Dev: 39.65252 IU/L] (Table 1).

Every weight rats group initially was compared with other one from 3 remained groups applying statistical paired t-test. (Table 2). Any emerging significant difference among ALP will be investigated whether owed in the above mentioned significant weight correlations. Every ALP rats group initially was compared with other one from 3 remainder groups applying statistical paired t-test (Table 2).

Table 3. The original influence of $\mathrm{U}-74389 \mathrm{G}$ in connection with reperfusion time

\begin{tabular}{|c|c|c|c|c|}
\hline & & & \multicolumn{2}{|c|}{ p-values } \\
\hline Increase (IU/L) & $95 \%$ c. in (IU/L) & Reperfusion time (h) & t-test & glm \\
\hline 10.6 & $-45.29532-66.49532$ & 1 & 0.6983 & 0.6950 \\
\hline 22.45 & $-56.3949-11.4949$ & 1.5 representative endpoint & 0.1733 & 0.1835 \\
\hline 34.3 & $-4.713154-73.31315$ & 2 & 0.0917 & 0.0812 \\
\hline
\end{tabular}


Table 4. The \% original alteration influence of U-74389G in connection with reperfusion time increasing

\begin{tabular}{lccl} 
Alteration & $\mathbf{\pm S D}$ & Reperfusion time $(\mathbf{h})$ & p-value \\
\hline$+7.75 \%$ & $20.87 \%$ & 1 & 0.6966 \\
$+19.76 \%$ & $+15.06 \%$ & 1.5 representative endpoint & 0.1784 \\
$+31.77 \%$ & $+18.43 \%$ & 2 & 0.0864 \\
$-25.21 \%$ & $+14.83 \%$ & reperfusion time & 0.0675 \\
$+8.08 \%$ & $+9.19 \%$ & interaction & $0.3696 \mathrm{~T}$ \\
\hline
\end{tabular}

\section{Results}

Applying generalised linear models (glm) with dependant variable the ALP levels and independent variables the U-74389G administration or no, the reperfusion time and their interaction, resulted in: $\mathrm{U}-74389 \mathrm{G}$ administration non-significantly increased the ALP levels by $22.45 \mathrm{IU} / \mathrm{L}$ [-11.09699 IU/L -55.99699 IU/L] $(\mathrm{p}=0.1835)$. This finding was in accordance with the results of paired t-test $(\mathrm{p}=0.1733)$. Reperfusion time non-significantly decreased the ALP levels by $28.65 \mathrm{IU} / \mathrm{L}$ [-61.68381 IU/L -4.38381 IU/L] (p=0.0872) also in accordance with paired t-test $(\mathrm{p}=0.0478)$. However, U-74389G administration and reperfusion time together kept non-significantly increased the ALP levels by $9.190909 \mathrm{IU} / \mathrm{L}$ [-11.30035 IU/L -29.68217 IU/L] ( $p=0.3696)$. Reviewing the above and Table 2, the Tables 3 and 4 sum up concerning the increasing influence of U-74389G in connection with reperfusion time. Inserting the rats weight also as an independent variable at generalised linear models analysis, a very significant relation results in ALP levels $(\mathrm{p}=0.0000)$, so as to further investigation is needed. The predicted ALP values adjusted for rats weight were calculated and are depicted at Table 5 .

\begin{tabular}{lcc} 
Table 5. Mean predicted ALP values adjusted for weight and \\
& Standard deviation of groups & \\
Groups & Mean (IU/L) & Std. Dev (IU/L) \\
\hline A & 111.8473 & 42.90819 \\
B & 94.03806 & 29.15938 \\
C & 140.4357 & 16.71637 \\
D & 142.779 & 16.96994 \\
\hline
\end{tabular}

The differences between predicted mean ALP values as calculated by paired t-tests are depicted at Table 6 . Applying generalised linear models with dependant variable the predicted ALP levels and independent variables the U-74389G administration or no, the reperfusion time and their interaction, resulted in: U-74389G administration significantly increased the predicted ALP levels by 38.66468 IU/L [20.39711 IU/L -56.93225 IU/L] $(p=0.0001)$. This finding was in accordance with the result of paired t-test $(\mathrm{p}=0.0002)$. Reperfusion time non-significantly decreased the predicted ALP levels by $7.732941 \mathrm{IU} / \mathrm{L}$ [-29.83455 IU/L $-14.36867 \mathrm{IU} / \mathrm{L}](\mathrm{p}=0.3926)$ also in accordance with paired t-test $(\mathrm{p}=0.3375)$. However, U-74389G administration and reperfusion time together significantly increased the predicted ALP levels by 21.51588 IU/L [10.11199 IU/L -32.91978
Table 6. Statistical significance of mean values difference for groups after statistical paired $t$ test application

\begin{tabular}{lcc} 
DG & Difference (IU/L) & p-value \\
\hline A-B & 17.8092 & 0.2423 \\
A-C & -28.58842 & 0.0674 \\
A-D & -30.93174 & 0.0574 \\
B-C & -46.39762 & 0.0019 \\
B-D & -48.74094 & 0.0004 \\
C-D & -2.343317 & 0.7043 \\
\hline
\end{tabular}

IU /L] ( $\mathrm{p}=0.0005)$. Reviewing the above and Table 6, the Tables 7 and 8 sum up concerning the increasing influence of $\mathrm{U}-74389 \mathrm{G}$ in connection with reperfusion time.

\section{Discussion}

ALP is being considered a reliable not only special tissue function but also general metabolic index. Its production is being influenced by ischemia and particulary by certain mode, as the next references show. Seifert et al. (2008) did not write down significant toxic ALP changes in subjects who ingested 20 grams/day of ribose with serial measurements by day 14 . Burlacu et al. (2008) investigated the extent of conversion in an attempt to increase the myogenic commitment of bone marrow stromal cells (BMSC) isolated from the adult rat tibia. The results revealed that cultured cells lost the native expression of ALP with time. Notably, the myogenic differentiation and particularly priming of mesenchymal cells to cardiomyogenic differentiation takes place over a short period of time. Hahn et al. (2007) submitted male Wistar rats into liver IR with or without ischemic precondition (IP). During IR, intraoperative radiotherapy (IORT) doses of 0-50 Gy were applied to the ischemic liver lobe. Shortand long-term ALP were significantly milder in groups with IP, especially those that were irradiated during ischemia. Irradiation during ischemia is less toxic for the liver tissue. Coux et al. (2002) examined the effects of IR on renal ALP activity in rats which showed biochemical alterations of brush border integrity, as suggested by compromised ALP excretion in urine. Koyama et al. (1998) showed differences in enzyme activities between arteriolar capillary portions who express ALP. Through use of ALP, adaptive changes in the capillary network were studied in rat hearts exposed to cold, exercise, hypertension, chronic coronary occlusion, and transient coronary occlusion followed by reperfusion. The increase in the arteriolar capillary portion seemed to be, however, a compensation for the limited angiogenesis. Doty et al. (1990) studied the effects of spaceflight on metaphyseal and cortical bone of the rat tibia in a spaceflight in 3-monthold male rats. The endosteal osteoblast population and the vasculature near the periosteal surface in flight rats showed more pronounced changes in cortical bone but no difference in the cell membrane-associated ALP activity than in metaphyseal bone compared with ground controls. Ansermet et al. (1980) measured the urinary excretion of enzyme ALP

Table 7. The predicted influence of U-74389G in connection with reperfusion time

\begin{tabular}{|c|c|c|c|c|}
\hline & & & \multicolumn{2}{|c|}{ p-values } \\
\hline Increase (IU/L) & $95 \%$ c. in (IU/L) & Reperfusion time (h) & t-test & glm \\
\hline 28.58842 & $-2.005433-59.18227$ & 1 & 0.0674 & 0.0653 \\
\hline 38.66468 & $20.39711-56.93225$ & 1.5 representative endpoint & 0.0002 & 0.0001 \\
\hline 48.74094 & 26.32647-71.15541 & 2 & 0.0004 & 0.0002 \\
\hline
\end{tabular}


Table 8. The \% predicted alteration influence of U-74389G in connection with reperfusion time

\begin{tabular}{lccl} 
Alteration & \pm SD & Reperfusion time $(\mathbf{h})$ & p-value \\
\hline$+22.66 \%$ & $+12.37 \%$ & 1 & 0.0663 \\
$+31.91 \%$ & $+7.69 \%$ & 1.5 representative endpoint & 0.0001 \\
$+41.16 \%$ & $+9.65 \%$ & 2 & 0.0003 \\
$-6.38 \%$ & $+9.30 \%$ & reperfusion time & 0.3650 \\
$+17.75 \%$ & $+4.79 \%$ & interaction & 0.0005 \\
\hline
\end{tabular}

in unanesthetized adult male Wistar rats within $48 \mathrm{~h}$ after either a single injection of mercuric chloride $\left(\mathrm{HgCl}_{2}\right)$, or of gentamicin, or of tobramoycin, or after $30 \mathrm{~min}$ of clamping of both renal arteries. The excretion of ALP was increased significantly above control levels after renal ischemia or the nephrotoxic agents; the increase was dose-related after $\mathrm{HgCl}_{2}$. Short-term exposure (3 months) to phenylmercuric acetate was associated with a significant decrease of the urinary excretion of ALP and of ALP activity.

U-74389G administration and its interaction with reperfusion time has non-significant increasing short-term effects on ALP levels. However, the predicted ALP values adjusted for rats weight, have significant increasing ones. It seems that U-74389G itself exerts a restorating influence on ALP levels during liver IR injury. The predicted results as more reliable show that the real restorating effect of $\mathrm{U}-74389 \mathrm{G}$ is slower than it seems, since rising from baseline ALP levels is significant within time context of 2 hours.

Acknowledgment: This study was funded by Scholarship by the Experimental Research Center ELPEN Pharmaceuticals (E.R.C.E), Athens, Greece. The research facilities for this project were provided by the aforementioned institution.

\section{REFERENCES}

Ansermet, F., Mieville, C., Diezi, J., 1980. Urinary enzyme excretion and changes in renal functions induced by toxic substances or by renal ischemia in rats. Arch. Toxicol. 4, 201-207.

Burlacu, A., Rosca, A.M., Maniu, H., Titorencu, I., Dragan, E., Jinga, V., Simionescu, M., 2008. Promoting effect of 5-azacytidine on the myogenic differentiation of bone marrow stromal cells. Eur. J. Cell. Biol. 87, 173-184.

Coux, G., Trumper, L., Elías, M.M., 2002. Renal function and cortical (Na(+)+K(+))-ATPase activity, abundance and distribution after ischaemia-reperfusion in rats. Biochim. Biophys. Acta. 2, 71-80.

Doty, S.B., Morey-Holton, E.R., Durnova, G.N., Kaplansky, A.S., 1990. Cosmos 1887: Morphology, histochemistry, and vasculature of the growing rat tibia. FASEB J. 4, 16-23.

Fenglin, S., Jennifer C., Kenneth L.A., 1995. 21-aminosteroid and 2-(aminomethyl) chromans inhibition of arachidonic acid-induced lipid peroxidation and permeability enhancement in bovine brain microvessel endothelial cell monolayers. Free Radic. Biol. Med. 19, $349-357$.

Hahn, O., Szijártó, A., Lotz, G., Schaff, Z., Vígváry, Z., Váli, L., Kupcsulik, P.K., 2007. The effect of ischemic preconditioning prior to intraoperative radiotherapy on ischemic and on reperfused rat liver. J. Surg. Res. 142, 32-44.

Koyama, T., Xie, Z., Gao, M., Suzuki, J., Batra, S., 1998. Adaptive changes in the capillary network in the left ventricle of rat heart. Jpn J. Physiol. 48, 229-241.

Seifert, J., Frelich, A., Shecterle, L., St Cyr, J., 2008. Assessment of hematological and biochemical parameters with extended D-Ribose ingestion. J. Int. Soc. Sports Nutr. 15, 5, 13. doi: 10.1186/1550-2783-5-13. 